\title{
Abnormal Retinal Vascular Reactivity in Individuals with Impaired Glucose Tolerance: A Preliminary Study
}

\author{
Sunni R. Patel, ${ }^{1}$ Srikanth Bellary, ${ }^{2}$ Lu Qin, ${ }^{1}$ George M. Balanos, ${ }^{3}$ David McIntyre, ${ }^{3}$ \\ and Doina Gherghel ${ }^{1}$
}

Purpose. To investigate the relationship between vascular function parameters measured at the retinal and systemic level and known markers for cardiovascular risk in patients with impaired glucose tolerance (IGT).

Methods. Sixty age- and sex-matched white European adults (30 with IGT and 30 with normal glucose tolerance [NGT]) were recruited for the study. Fasting plasma glucose, lipids, and 24-hour blood pressure (BP) were measured in all subjects. Systemic vascular and endothelial function was assessed by using carotid-artery intimal media thickness (cIMT) and flowmediated dilation (FMD). Retinal vascular reactivity was assessed by the dynamic retinal vessel analyzer. Additionally, blood glutathione (reduced glutathione [GSH], glutathione disulfide [GSSG], and total glutathione [tGSH]) and plasma von Willebrand (vWF) factor levels were also measured.

Results. Individuals with IGT demonstrated higher BP values ( $P$ $<0.001$ ), fasting triglyceride (TG) levels and TG:high-density lipoprotein (HDL) ratios $(P<0.001)$ than NGT subjects. Furthermore, total:HDL-cholesterol (HDL-C) ratios and Framingham scores were raised $(P=0.010$ and $P<0.001$, respectively). Blood glutathione levels (GSH, GSSG, and tGSH) were lower $(P<0.001, P=0.039$, and $P<0.001$, respectively) while plasma vWF was increased $(P=0.014)$ in IGT subjects compared to controls. IGT individuals also demonstrated higher IMT in right and left carotid arteries $(P=0.017$ and $P$ $=0.005$, respectively) alongside larger brachial artery diameter $(P=0.015)$ and lower FMD percentage $(P=0.026)$ and glyceryl trinitrate-induced dilation $(P=0.012)$ than healthy controls. At the retinal arterial level, the IGT subjects showed higher baseline diameter fluctuations (BDFs) $(P=0.026)$, longer reaction time $(\mathrm{RT})(P=0.032)$, and reduced baseline-corrected flicker response (bFR) $(P=0.045)$. In IGT subjects, retinal BDF correlated with total:HDL $(P=0.003)$ and HDL-C $(P=0.004)$. Arterial RT also correlated with FMD $(P=0.017)$ in IGT but not NGT subjects.

ConcLusions. In IGT individuals there is a relationship between macro- and microvascular function, as well as a direct correlation between the observed retinal microcirculatory changes and established plasma markers for cardiovascular

From the ${ }^{1}$ Vascular Research Laboratory, Ophthalmic Research Group, School of Life and Health Sciences; the ${ }^{2}$ Aston Research Centre for Healthy Ageing, Aston University, Birmingham, United Kingdom; and the ${ }^{3}$ School of Sport and Exercise Sciences, University of Birmingham, United Kingdom.

Submitted for publication January 16, 2012; revised May 2 and May 29, 2012; accepted June 23, 2012.

Disclosure: S.R. Patel, None; S. Bellary, None; L. Qin, None; G.M. Balanos, None; D. McIntyre, None; D. Gherghel, None

Corresponding author: Doina Gherghel, Vascular Research Laboratory, School of Life and Health Sciences, Aston University, Birmingham, B4 7ET, UK; d.gherghel@aston.ac.uk. diseases. Multifactorial preventive interventions to decrease vascular risk in these individuals should be considered. (Invest Ophthalmol Vis Sci. 2012;53:5102-5108) DOI:10.1167/ iovs.12-9512

mpaired glucose tolerance (IGT) refers to an intermediate 1 metabolic state between normal and diabetic state and is characterized by insulin resistance and impairment in insulin secretion. It is predicted that the numbers of those diagnosed with IGT worldwide will rise from 200 million at present to 400 million by the year $2030 .{ }^{1}$ A proportion (5\%-12\%) of those diagnosed with IGT will progress to type 2 diabetes mellitus (T2DM) each year, therefore representing a major cause of public health concern. IGT is also associated with a high risk of cardiovascular disease (CVD), ${ }^{2}$ and this, along with being a transition stage towards T2DM, qualifies this condition as a disease state. ${ }^{3}$ Consequently, close follow-up of individuals suffering from IGT, and early identification of vascular dysfunction, could represent an important step in prevention of future cardiovascular morbidities.

Recent evidence suggests that endothelial dysfunction, an early pathophysiologic stage in the development of T2DM and/ or CVD, can be detected very early in subjects at risk, often before the manifestation of carbohydrate intolerance. Indeed, IGT subjects have been shown to demonstrate both systemic macrovascular endothelial dysfunction and functional changes in the microvasculature similar to those observed in patients with early T2DM. ${ }^{4,5}$ This latter observation is particularly important owing to supporting evidence suggesting that the microvasculature is an early target for systemic diseases such as T2DM. Consequently, measurements that identify early changes at this level could potentially be used as predictors of local or systemic vascular complications. ${ }^{6}$

Dynamic retinal vessel analysis, a noninvasive technique that assesses retinal vascular responses to flickering light, has been shown to identify early signs of vascular dysfunction in individuals with various degrees of risk for CVD even in the absence of overt clinical disease. ${ }^{7,8}$ Indeed, by using this technique, we have demonstrated that young, healthy individuals with a genetic predisposition towards diabetes exhibit early signs of impaired retinal dilatory responses. ${ }^{7}$

Previous research has shown early abnormal microvascular vasodilation alongside macrovascular changes in individuals at risk for T2DM. ${ }^{9}$ However, a correlation between micro- and macrovascular reactivity measures is found only when the study groups (controls, relatives of T2DM patients, IGT individuals, and T2DM patients) are grouped together. Consequently, as no other studies have addressed this issue, to date there is still very little knowledge about simultaneous vascular function changes at both the micro- and macrocirculation level and their importance in individuals with other predisposing risk factors for T2DM, such as IGT. Therefore, the aim of this study was to investigate the relationship between vascular 
function parameters measured at the retinal and systemic level and known markers for CVD risk in patients with IGT.

\section{Methods}

\section{Study Sample}

White European subjects aged 35 to 55 years were recruited through a nurse-led community oral glucose tolerance test (OGTT) clinic within the Birmingham East and North National Health Service (NHS) Trust, Birmingham, United Kingdom, from October 2010 to April 2011. Subjects were referred by their primary care physician for suspected glucose metabolism issues (i.e., suspected T2DM or IGT). A 75-g OGTT was carried out as per World Health Organization protocol and a diagnosis of IGT was confirmed by a consultant diabetologist. ${ }^{10}$ All IGT subjects were considered "patient-naïve," as they were unaware of the IGT diagnosis at the time of the OGTT. The investigator (S.R.P.) was masked from the OGTT, and thus there was no bias in patient selection, that is, only selecting "high-risk" subjects. In addition, age-matched normoglycemic controls (also confirmed after OGTT) were recruited through promotion of the study at the Aston University Health Clinics, Birmingham, United Kingdom.

Subjects were excluded if they had a positive diagnosis or were taking medication for cardio- or cerebrovascular disease, coronary artery disease, heart failure, arrhythmia, stroke, transient ischemic attacks, peripheral vascular disease, hypertension, severe dyslipidemia (defined as plasma TG $>6.00 \mathrm{mM}$ or cholesterol levels $>7.00 \mathrm{mM}$ ), and diabetes or were smokers (including ex-smokers). All subjects taking vasoactive substances, including dietary/vitamin/antioxidant supplementation and bronchodilators, were not included in the present study. In addition, subjects were also excluded if they had a refractive error of more than \pm 3 diopter spheres and more than \pm 1 diopter cylindrical equivalent, an intraocular pressure (IOP) of more than $24 \mathrm{~mm} \mathrm{Hg}$, cataract, or any other media opacities that either prevent retinal vascular examination or undermine the quality of data, as well as if they had a history of intraocular surgery or any form of retinal or neuroophthalmic disease affecting the ocular vascular system.

Written informed consent was obtained from all participants and ethical approval was sought from local and NHS ethical committees. The study was designed and conducted in accordance with the tenets of the Declaration of Helsinki.

\section{Investigations}

All the participants underwent a routine ophthalmologic assessment and standardized anthropometric measurements for height and weight

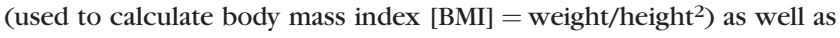
waist and hip measurements (used to calculate waist to hip ratio). Female participants were asked to fill in a validated menstrual cycle questionnaire and their investigations were carried out during the first week of the menstrual cycle (follicular phase) as per previously established protocol. ${ }^{11,12}$

\section{Blood Sampling and Analyses}

General Assessment for CVD Risk. All participants were asked to fast and refrain from caffeine, alcohol, chocolate, and carbonated drinks and to not exercise for 12 hours before the date of the study. All blood samples were obtained by a qualified phlebotomist in the morning, between $9 \mathrm{AM}$ and $10 \mathrm{AM}$. Fasting plasma glucose, TG, total cholesterol, and HDL-C were measured by using standard routine laboratory techniques with the Reflotron Desktop Analyzer (Roche Diagnostics, UK). The TG:HDL-C ratio, a measure of endothelial function, and the Framingham score as a means of cardiovascular risk for a 10 -year period, ${ }^{13}$ were also determined from the above values.

Oxidative Stress Markers. Fasted blood was collected and initial processing was carried out within 10 minutes of blood collection. Each sample was then processed and analyzed for reduced glutathione (GSH), glutathione disulfide (GSSG), and total glutathione (tGSH), according to previous protocols. ${ }^{14}$

\section{Vascular Endothelial Cell Function Markers.}

Von Willebrand Factor ( $v W F$ ) Assay. Fasted blood was collected by venepuncture and processed for further batch analysis by enzymelinked immunosorbent assay according to an established protocol. ${ }^{15}$

\section{Ambulatory Blood Pressure (BP) Measurements}

Systemic BP was measured by using a 24-hour computer-operated ambulatory BP monitor (Cardiotens-01; Meditech Ltd., Hungary) for each subject. Mean arterial pressure (MAP), as a means of describing cardiac output function in relation to arteriolar resistance, was calculated by using the following equation: $\mathrm{MAP} \approx(2 / 3 \times \mathrm{DBP})+$ $(1 / 3 \times \mathrm{SBP})$ with the pulse pressure $(\mathrm{PP})$ parameter equation: $\mathrm{PP}=\mathrm{SBP}-$ DBP, where SBP is systolic BP and DBP is diastolic BP.

\section{Intima-Media Thickness (IMT) Assessment}

High-resolution B-mode ultrasound system (Acuson Sequoia, 5-MHz linear transducer; Siemens) was used to obtain longitudinal images of the right and left extracranial far wall of the common carotid artery (CCA). The images were evaluated by using ultrasonographic frames of the CCA as previously recommended. ${ }^{16}$ In each carotid segment, farwall IMT was measured bilaterally for a single CCA view. Each IMT measure, also used for statistical analysis, represented an average of three to five measurement points.

\section{Vascular Function Studies}

Retinal vessel reactivity was measured with the dynamic retinal vessel analyzer (DVA, IMEDOS; GmbH, Jena, Germany) by using an already established and recommended protocol. ${ }^{17,18}$ All measurements were performed in one randomly selected eye for each subject, between 8:00 and 11:00 $\mathrm{AM}$ in a quiet, temperature-controlled room $\left(22^{\circ} \mathrm{C}\right)$. Following full-pupil dilation with tropicamide 1\% (Minims; Chauvin Pharmaceuticals Ltd., UK), a region of interest encompassing vessel segments of approximately $500 \mu \mathrm{m}$ was chosen. Retinal vessel reactivity, in the form of arterial and venous reaction time (RT) and maximum diameter (MD), were determined with our newly defined method of sequential and diameter response analysis (SDRA), which is described elsewhere. ${ }^{19}$ In short, SDRA tries to overcome what were considered as shortfalls of the DVA software algorithms and focuses on time and diameter responses for each flicker response as opposed to an averaged response. Furthermore, the fluctuations in baseline diameter are taken into account, thus providing further insight into reaction patterns as a possible tool for the assessment of vessel physiology. ${ }^{19}$ The following retinal vessel reactivity and time course parameters were calculated: baseline diameter fluctuation (BDF) as the difference between maximum and minimum baseline vessel diameter; the MD as the maximal vessel dilation in response to flicker light stimulation expressed as a percentage from baseline; the MD reaction time (MDRT) as the time taken (seconds) to reach the maximum vessel diameter during 20-second flicker exposure; the maximum constriction (MC) as the percentage to baseline minimal vessel diameter within 30 seconds of the recovery period; and the maximum constriction reaction time (MCRT) as the time taken (seconds) to reach maximal vessel constriction. In addition, the dilation amplitude, as the difference between maximal dilation and constriction responses and the baselinecorrected flicker response (bFR), which accounted for the BDF, were also computed.

Brachial artery flow-mediated dilation (FMD) was measured by using high-resolution color Doppler imaging ultrasonography, with a 7$\mathrm{mm}$ 8-MHz linear-array (Acuson Sequoia; Siemens, UK), and the diameter was continuously measured by using wall-detection specialized artificial neural networking software (VIA Software, UK) from the anterior to the posterior interface between the media and adventitia on 
TABLE 1. Baseline Demographic and Metabolic Data of Both Groups

\begin{tabular}{|c|c|c|c|}
\hline & NGT $(n=30)$ & IGT $(n=30)$ & $P$ Value \\
\hline Demographic data & $15 \mathrm{M}: 15 \mathrm{~F}$ & $15 \mathrm{M}: 15 \mathrm{~F}$ & \\
\hline Age, y & $47.7 \pm 10$ & $47.7 \pm 10.2$ & - \\
\hline Weight, kg & $75.6 \pm 14.7$ & $80.2 \pm 14.6$ & 0.127 \\
\hline BMI, $\mathrm{kg} / \mathrm{m}^{2}$ & $26.0 \pm 4.22$ & $27.6 \pm 5.33$ & 0.092 \\
\hline WHR & $0.93 \pm 0.11$ & $0.97 \pm 0.05$ & 0.075 \\
\hline $\mathrm{SBP}, \mathrm{mm} \mathrm{Hg}$ & $118 \pm 12$ & $127 \pm 18^{*}$ & 0.001 \\
\hline DBP, mm Hg & $75 \pm 8$ & $80 \pm 11^{*}$ & 0.010 \\
\hline MAP, mm Hg & $89 \pm 10$ & $96 \pm 13^{*}$ & 0.004 \\
\hline $\mathrm{PP}, \mathrm{mm} \mathrm{Hg}$ & $43 \pm 10$ & $47 \pm 10^{*}$ & 0.010 \\
\hline IOP, $\mathrm{mm} \mathrm{Hg}$ & $14 \pm 3$ & $15 \pm 2$ & 0.090 \\
\hline \multicolumn{4}{|l|}{ Metabolic data } \\
\hline Fasting glucose, mM & $4.84 \pm 0.69$ & $5.46 \pm 0.84^{*}$ & $<0.001$ \\
\hline 2-h GTT, mM & $6.06 \pm 1.03$ & $9.81 \pm 2.09^{*}$ & $<0.001$ \\
\hline $\mathrm{TG}, \mathrm{mM}$ & $1.20 \pm 0.51$ & $1.76 \pm 1.02^{*}$ & $<0.001$ \\
\hline HDL-C, mM & $1.21 \pm 0.38$ & $1.08 \pm 0.40$ & 0.120 \\
\hline LDL cholesterol, mM & $2.65 \pm 0.77$ & $2.67 \pm 1.03$ & 0.883 \\
\hline Total cholesterol, $\mathrm{mM}$ & $4.39 \pm 0.82$ & $4.56 \pm 1.06$ & 0.372 \\
\hline TG:HDL-C, mM & $2.59 \pm 1.62$ & $4.81 \pm 4.36^{*}$ & $<0.001$ \\
\hline \multicolumn{4}{|l|}{ CVD risk data } \\
\hline Total:HDL-C, mM & $3.98 \pm 1.33$ & $4.78 \pm 2.17^{*}$ & 0.010 \\
\hline R-IMT, mm & $0.055 \pm 0.015$ & $0.065 \pm 0.015^{*}$ & 0.010 \\
\hline L-IMT, mm & $0.057 \pm 0.017$ & $0.069 \pm 0.016^{*}$ & 0.005 \\
\hline Framingham score & $1.9 \pm 2.8$ & $4.5 \pm 5.8^{*}$ & $<0.001$ \\
\hline
\end{tabular}

Values quoted in mean \pm SD. GTT, glucose tolerance test; L-IMT, left intima media thickness; R-IMT, right intima media thickness; WHR, waist to hip ratio.

* Significant differences indicated in bold as $P \leq 0.01$.

a personal computer. The procedure was carried out to a previously validated and universally accepted protocol of 2 minutes baseline, followed by 5 minutes occlusion and 2 minutes recovery. Following 10 minutes of reacclimatization, an exogenous nitric oxide donor (300 mcg sublingual glyceryl trinitrate [GTN] tablet) was administered. The FMD percentage was determined as absolute change from baseline, as well as GTN-induced changes, and a ratio of both of these values was then determined. ${ }^{20}$

\section{Power Calculation and Statistical Analysis}

From previous studies, a change of $30 \%$ with an SD of $2.5 \%$ in retinal vessel reactivity has been shown to be significant. ${ }^{21,22}$ Additionally, FMD studies have shown a $20 \%$ reduction with an SD of $1.85 \%$ in FMD response in those with IGT. ${ }^{23}$ As the study design was multifactorial in nature, it was calculated that $n=25$ was sufficient to provide $90 \%$ power with an $\alpha$ of 0.05 . Furthermore, the sensitivity and reproducibility of the techniques in healthy subjects have been reported previously. ${ }^{12,24}$

All analyses were performed with Statistica software (version 9; StatSoft Inc., Tulsa, OK). Before all analysis, data were tested for normal distribution and thus a suitable test was adopted. Differences in mean values for each of the measured variables were compared by independent samples $t$-test (or Wilcoxon) for continuous variables. A multivariate analysis was performed to investigate possible influences of parameters such as age, sex, BMI, BP, and circulating biomarkers (such as glucose, TG, cholesterol, vWF, and glutathione levels) on the measured variables. Comparison of retinal vessel reactivity for each flicker period was made by repeated measures (or Friedman) analysis of variance (ANOVA) following within-group analysis. Differences between groups in retinal and systemic vascular function were computed by analysis of covariance (ANCOVA) where applicable. A $P$ value of $<0.05$ was considered statistically significant, unless stricter criteria were adopted for within-group and multivariate analysis $(P \leq$ 0.01 to account for multiple comparisons and thereby minimize bias towards type II errors).

\section{Results}

An initial 100 individuals were screened at a nurse-led clinic, and following OGTT and confirmed IGT diagnosis, 40 were excluded as they did not meet the inclusion criteria (preexisting comorbidity and/or taking vasoactive medication; 20 diagnosed with T2DM, 10 with cardiovascular issues, and 10 presenting with gestational diabetes mellitus). Finally, 30 White European IGT subjects (15 men and 15 women) and 30 normal glucose tolerance (NGT) subjects (15 men and 15 women) were recruited for the final protocol.

The baseline characteristics of both groups are presented in Table 1 . There were no significant differences in age, IOP, and cholesterol levels (HDL, low-density lipoprotein [LDL], and total cholesterol) between IGT subjects and controls. However, compared to healthy age- and sex- matched NGT controls, the IGT group had significantly higher 24-hour SBP, DBP, MAP, and PP values $(P=0.001, P=0.017, P=0.004$, and $P=0.023$, respectively), alongside raised fasting levels of glucose and TG $(P<0.001)$. TG:HDL-C and total:HDL-C ratios, as well as Framingham scores, were also significantly higher in those with IGT $(P<0.001, P=0.010$, and $P<0.001$, respectively) than in NGT healthy controls. Those with IGT showed greater right- $(P=0.017)$ and left-side $(P=0.005)$ carotid artery IMT than their normoglycemic counterparts.

IGT subjects had lower blood GSH $(P<0.001)$, GSSG $(P=$ 0.039), and tGSH $(P<0.001)$, as well as higher plasma vWF levels $(P=0.014)$, than the NGT subjects (Table 2$)$. The average retinal arterial $\mathrm{BDF}(P=0.026)$ and MDRT $(P=0.032)$ were higher and bFR was lower $(P=0.045)$ in IGT individuals than in NGT subjects (Table 3). Figures 1 and 2 show the individual three flicker cycle responses and relevant significant $P$ values for MDRT (flicker cycle $1, P=0.011$; and flicker cycle $2, P=0.022$ ) and bFR (flicker cycle $2, P=0.014$ ). Multiple regression analyses showed significant correlations between vWF and arterial RT $(P=0.029)$ only; therefore, the $P$ values quoted were calculated with ANCOVA correcting for these significant confounding variables.

In addition, a multivariate analysis showed significant and positive correlations between retinal arterial BDF and total:HDL $(r=0.541 ; P=0.003)$, as well as negative correlation

TABLE 2. Plasma Markers for Oxidative Stress and Endothelial Function in Both Groups

\begin{tabular}{lcrr}
\hline & NGT $(\boldsymbol{n}=\mathbf{3 0})$ & IGT $(\boldsymbol{n}=\mathbf{3 0})$ & $\boldsymbol{P}$ Value \\
\hline GSH, $\mu$ M & $934.33(407.15-1293.81)$ & $536.09(300.19-688.52)^{*}$ & $<\mathbf{0 . 0 0 1}$ \\
GSSG, $\mu$ M & $98.11(40.93-117.27)$ & $64.53(26.84-68.40)^{*}$ & $\mathbf{0 . 0 3 9}$ \\
GSH:GSSG & $15.25(5.37-21.95)$ & $13.05(6.05-18.83)$ & 0.311 \\
tGSH, $\mu$ M & $1130.54(592.74-1634.95)$ & $665.15(399.48-761.24)^{*}$ & $<\mathbf{0 . 0 0 1}$ \\
vWF, $\mu$ dL & $122.3 \pm 54.9$ & $149.8 \pm 59.2^{*}$ & $\mathbf{0 . 0 1 4}$
\end{tabular}

* Significant differences indicated in bold as $P<0.05$. 
TABLE 3. Average Retinal Arterial and Venous Measures for Both Groups

\begin{tabular}{lccc}
\hline & NGT $(\boldsymbol{n}=30)$ & \multicolumn{1}{c}{ IGT $(\boldsymbol{n}=\mathbf{3 0})$} & P Value \\
\hline Artery & & & \\
AD, $\mu$ M & $123.94(112.85-133.77)$ & $121.61(96.91-174.28)$ & 0.501 \\
BDF, AU & $5.14(3.47-6.44)$ & $6.42(1.57-13.16)^{*}$ & $\mathbf{0 . 0 2 6}$ \\
MD, \% & $5.05(3.18-6.37)$ & $5.48(1.17-10.3)$ & 0.433 \\
MDRT, s & $18.4(13.3-22.7)$ & $21.4(10.7-37.0)^{*}$ & $\mathbf{0 . 0 3 2}$ \\
MC, \% & $3.24(1.84-4.29)$ & $2.96(0.45-6.48)$ & 0.546 \\
MCRT, s & $20.1(17.3-23.3)$ & $19.7(8.3-29.0)$ & 0.691 \\
DA, \% & $8.27(5.99-9.73)$ & $8.44(2.19-16.47)$ & 0.820 \\
bFR, \% & $3.13(1.48-4.75)$ & $2.01(-5.57 \text { to } 7.17)^{*}$ & $\mathbf{0 . 0 4 5}$ \\
Vein & & & \\
AD, AU & $155.75(142.16-170.29)$ & $159.29(124.70-202.27)$ & 0.468 \\
BDF, AU & $4.03(2.54-4.96)$ & $4.66(2.04-9.89)$ & 0.150 \\
MD, \% & $5.71(4.09-6.54)$ & $5.92(1.61-12.08)$ & 0.704 \\
MDRT, s & $19.5(17.0-22.0)$ & $21.7(12.0-31.7)$ & 0.053 \\
MC, \% & $1.43(0.40-1.84)$ & $2.01(0.95-10.15)$ & 0.114 \\
MCRT, s & $21.9(19.3-25.3)$ & $20.3(7.0-29.3)$ & 0.149 \\
DA, \% & $7.14(4.90-8.54)$ & $7.83(3.12-13.09)$ & 0.290 \\
bFR, \% & $3.12(1.57-4.30)$ & $3.14(-1.92$ to 10.76$)$ & 0.975 \\
\hline
\end{tabular}

Measures were corrected for age, sex, weight, BP, and circulating serum markers. $\mathrm{AD}$, absolute diameter; $\mathrm{AU}$, arbitrary units; $\mathrm{DA}$, dilation amplitude; MCRT, reaction time to maximum constriction post flicker; MD, maximum dilation; MDRT, reaction time to reach maximum diameter to flicker stimulation.

* Significant differences indicated in bold (ANCOVA, $P<0.05$ ).

with HDL-C $(r=-0.522 ; P=0.004)$ (Fig. 3). Furthermore, retinal arterial MDRT correlated negatively with brachial artery FMD $(r=-0.4546, P=0.017)$ in the IGT but not the NGT group (Fig. 4).

No significant correlations were found between the measured blood glutathione levels (GSH, GSSG, tGSH, and GSH:GSSG) and retinal vascular reactivity parameters (all $P>$ 0.05).

An ANCOVA analysis revealed that the IGT group showed a larger brachial artery diameter $(P=0.015)$ but lower FMD percentage than the control group $(P=0.026)$. Furthermore, the GTN-induced dilation was lower $(P=0.012)$ and the

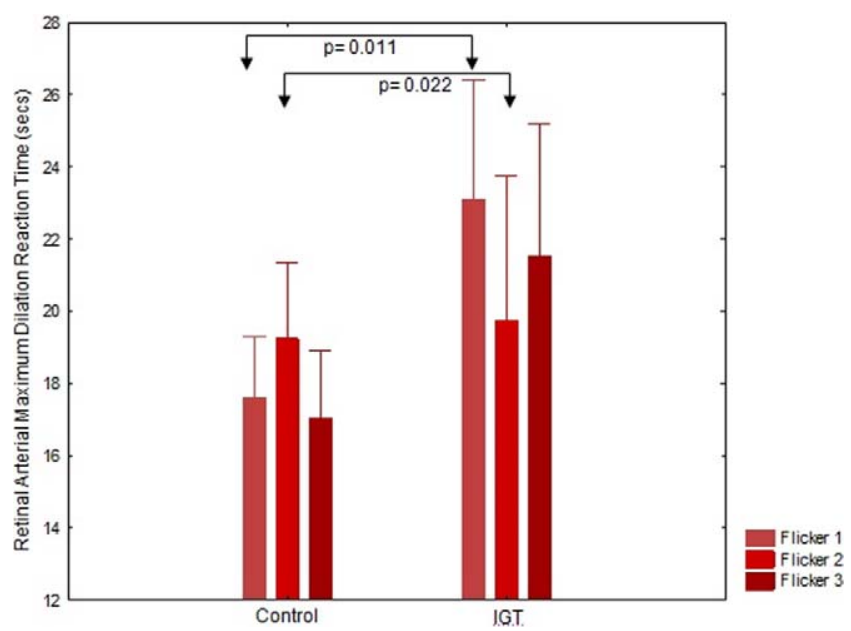

Figure 1. Arterial MDRT for each of the three flicker cycles when corrected for confounding variables (anthropometric: BP, and circulating markers: glucose, TG, TG:HDL) in the IGT group as compared to NGT controls. Bars: average MDRT for each individual flicker cycle. Error bars: SD of mean. Significant differences indicated in the first and second flicker cycles as shown by the $P$ values.

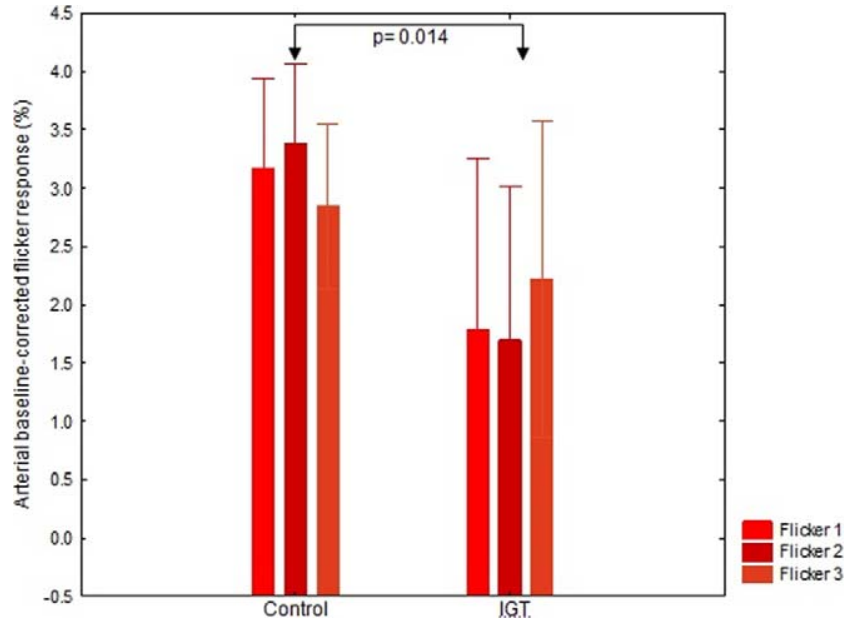

FIgURE 2. Arterial bFR when corrected for confounding variables for each of the three flicker cycles for the IGT group as compared to NGT controls. Bars: average bFR for each individual flicker cycle. Error bars: SD of mean. Post hoc analysis of re-ANOVA showed a significant reduction in bFR for the second flicker cycle.

FMD:GID ratio was higher $(P=0.034)$ in the IGT group than in healthy controls (Table 4).

\section{Discussion}

The results of our pilot study suggest that IGT individuals demonstrate signs of early vascular dysfunction as measured by functional (at macro- and microcirculatory levels) and circulatory markers. Moreover, in addition to a relationship between functional macro- and microvascular parameters, there appears to be a direct correlation between the observed retinal microcirculatory changes and established plasma markers for CVD risk.

Consistent with previous research, ${ }^{25,26}$ individuals with IGT recruited for this present study presented with high BP levels as well as increased markers of systemic endothelial dysfunction (impaired FMD response and increased vWF). They also demonstrated high levels of dyslipidemia (higher TG levels and cholesterol ratios) and Framingham risk scores. All these factors are well known to contribute towards CVD risk in prediabetes. ${ }^{27}$ In particular, dyslipidemia results in a reduced nitric oxide (NO) availability, possibly through generation of reactive oxygen species (ROS) that act to quench $\mathrm{NO}{ }^{28}$ Indeed, in line with previous research, ${ }^{29}$ our IGT group also showed reduced levels of blood glutathione levels (in reduced and oxidized forms) when compared to the NGT group. This observation, in association with the observed abnormal lipid metabolism, could be used to explain, to some extent, the observed presence of endothelial dysfunction at multiple levels in our IGT group. ${ }^{30}$ The relationship between circulatory markers for dyslipidemia and oxidative stress is, however, complex, and the contribution of these factors, separate or in combination to the onset and degree of vascular dysfunction measured in various vascular beds, should be assessed in future research.

It is well known that insulin resistance plays an important role in the development of endothelial dysfunction through reduced production of $\mathrm{NO}^{31}$ and accelerated atherosclerosis in IGT. ${ }^{32}$ Indeed, our IGT cohort demonstrated signs of early atherosclerotic changes as measured by increased IMT at the carotid level. This observation is in accordance with previous results, ${ }^{33,34}$ and, in the light of all the other reported changes, 

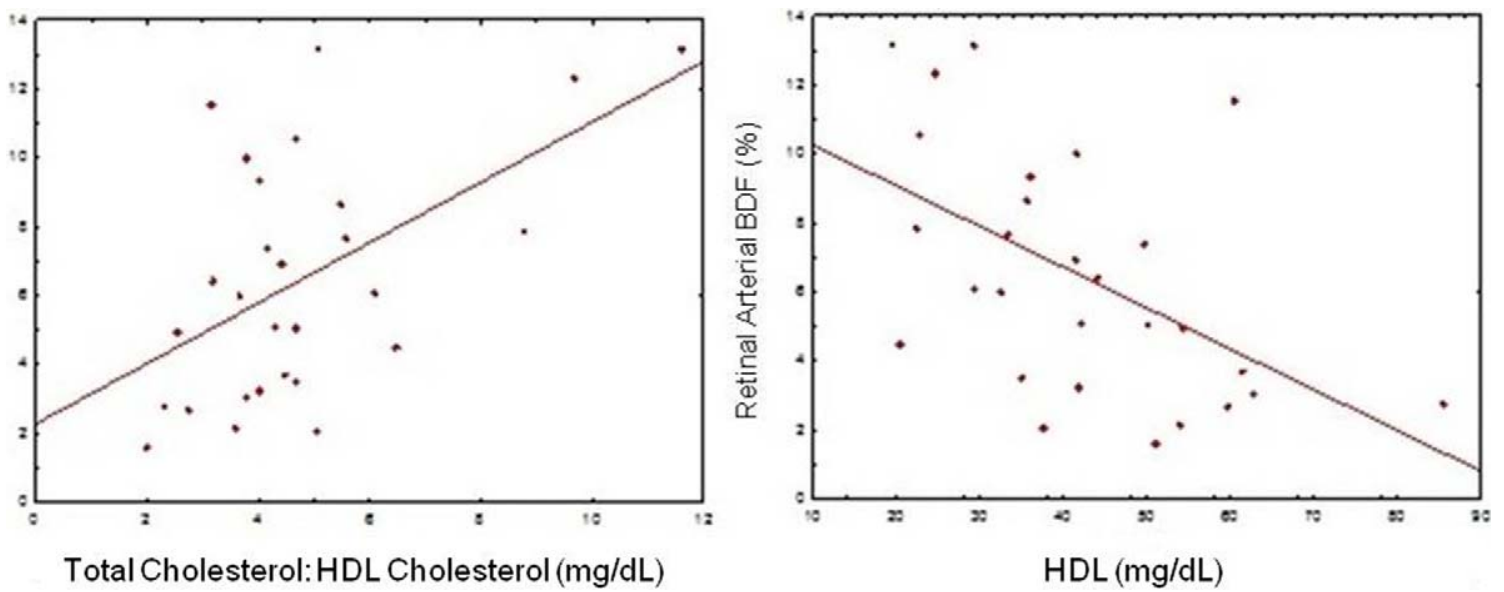

FIGURE 3. Lipid level and arterial BDF multivariate correlations in IGTs. Positive Spearman correlations for BDF against total cholesterol:HDL-C $(r=$ $0.541 ; P=0.003)$ and a negative correlation with arterial BDF against HDL-C $(r=-0.5222 ; P=0.004)$.

it possibly emphasizes the necessity of early cardiovascular screening and intervention in prediabetes status including IGT.

An important finding of the present study was the presence of impaired retinal vessel function to flicker (greater fluctuations in arterial BDF, increased MDRT, and a reduced bFR), as assessed by the DVA. Previous studies showing an increased risk for diabetic retinopathy in IGT, 35,36 have included individuals suffering from hypertension, which could have acted as a possible confounding factor. ${ }^{37}$ Moreover, the observed changes were structural only and associated with standard static fundus assessment, and to date, there is no research showing preclinical functional retinal vascular changes in subjects with IGT. Our study reported such changes for the first time and although the precise mechanisms behind our results need further elucidation, we can formulate some hypotheses. For instance, there is some evidence to suggest that an increased MDRT could be the result of either early atherosclerosis, increased arterial stiffness, or reduced NO bioavailability to peripheral tissues. ${ }^{7}$ Indeed, we have already shown that all these mechanisms could be implicated in our observed macrovascular changes; therefore, it is not unreasonable to presume that similar relationships also occur at the microvascular level including retinal microcirculation.

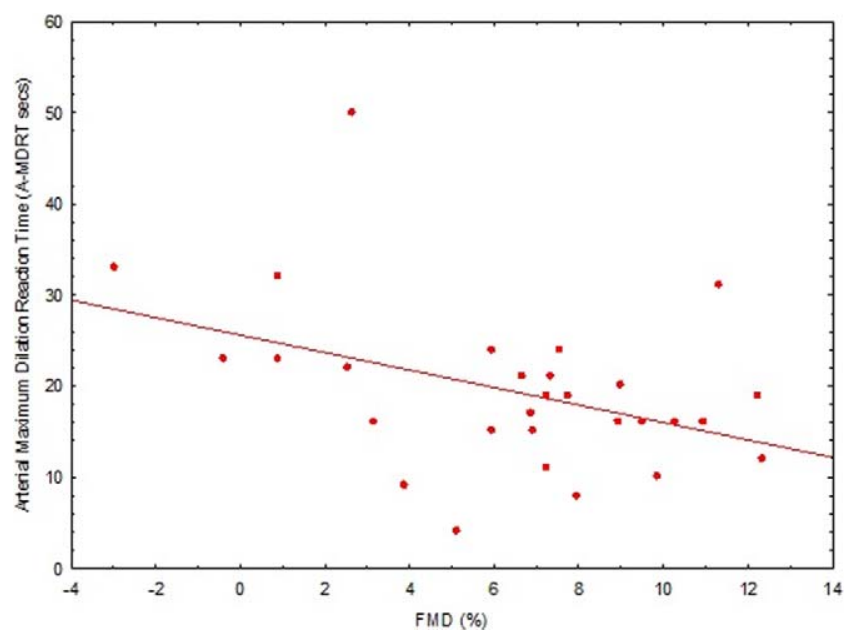

Figure 4. Negative average arterial MDRT multivariate correlation with brachial artery FMD in the IGT group (Spearman, $r=-0.4546, P=$ 0.017).
We also observed a higher fluctuation in retinal arterial BDF with IGT status. BDF is a parameter, which although recognized, is not commonly reported in the literature. It is reported to demonstrate the effect of spontaneous variations in vessel diameter that occur under normal resting conditions to flicker light. ${ }^{38}$ Tentative links have been made in previous studies between the occurrence of increased arterial diameter fluctuations and the presence of vascular disturbance in both smokers and vasospastic subjects ${ }^{19,39,40}$ and between increased retinal venous diameters and the presence of retinal ischemia and hypoxia. ${ }^{41,42}$ On this basis, it could be hypothesized that the increased BDF identified in our IGT patients may represent an indicator that common alterations in retinal vascular function may exist in these individuals, perhaps related to an increased variation in vascular tone or compliance. Indeed, it is important to emphasize here that in our IGT sample there was a correlation between signs of retinal vascular dysfunction and plasma lipid levels, possibly suggesting that dyslipidemia itself could have a direct influence on the retinal vascular response as assessed by the DVA. ${ }^{7}$

All the above hypotheses, however, would require further clarification and research. Nevertheless, another interesting observation was that our IGT individuals showed a correlation between abnormal retinal vascular reactivity and the validated measure of systemic endothelial dysfunction (FMD). This relationship could suggest that, although the macro- and

Table 4. Average Brachial Artery Reactivity as Means of Systemic Vascular Endothelial Function between Both Groups

\begin{tabular}{|c|c|c|c|}
\hline & $(n=30)$ & IGT $(n=30)$ & $P$ Value \\
\hline \multicolumn{4}{|l|}{ Brachial artery } \\
\hline $\begin{array}{l}\text { Baseline diameter, } \\
\mathrm{mm}\end{array}$ & $4.04(3.34-4.61)$ & $4.53(3.94-4.91)^{*}$ & 0.015 \\
\hline $\begin{array}{l}\text { Peak diameter, } \\
\text { mm }\end{array}$ & 4.40 & 4.7 & 119 \\
\hline $\begin{array}{l}\text { FMD, \% } \\
\text { GTN }\end{array}$ & $8.61(2.98-10.65)$ & $5.50(2.16-8.51)^{*}$ & .026 \\
\hline $\begin{array}{l}\text { Peak diameter, } \\
\text { mm }\end{array}$ & 5.02 & $5.27(4.6$ & 0.307 \\
\hline GID, \% & $25.20(17.35-31.69)$ & $16.28(14.16-19.27)^{*}$ & 0.012 \\
\hline
\end{tabular}

Reactivity was corrected for age, sex, weight, BP, and circulating serum markers. Values quoted in mean (IQR). GID, GTN-induced dilation.

* Significant differences indicated in bold (ANCOVA, $P<0.05$ ). 
microvascular beds' functions are governed by different physiological mechanisms, the pathologic changes associated with the IGT state could affect all circulatory beds in a similar manner. Indeed, it is well known that vascular pathologies occurring in a given tissue or organ are not isolated, and both the initiation and risk for further vascular dysfunction are triggered by common mechanisms acting similarly at both macro- and microcirculation levels. ${ }^{43}$ This is particularly important in prediabetes and diabetes in which generalized endothelial dysfunction can be induced by a combination of pathologic changes including dyslipidemia, hypertension, IR, and high levels of ROS, all mentioned above and measured in the present research.

The pilot design of the present study could be perceived as a limitation. The cross-sectional design of the study with a small cohort can therefore represent a drawback. The authors also recognize that future work would also need to incorporate further biochemical analyses, including HbA1c and fasting insulin levels, liver and kidney function tests, and hematology profiles, in order to best understand the glycemic and vascular function status at the time of diagnosis. Furthermore, the influences of other nondietary and social factors (including exercise) should be included in future work.

Nevertheless, the significant differences observed between those with IGT and NGT would suggest that early changes in vascular function can be seen in such individuals at both macro- and microcirculatory levels. In addition, the relationship between functional macro- and microvascular parameters, observed retinal microcirculatory changes, and established CVD risk markers emphasizes the importance of early vascular screening in IGT subjects. Retinal vascular imaging could emerge as a possible future option for individual risk stratification in diseased patients ${ }^{44,45}$ but also in individuals at risk for metabolic and cardiovascular pathologies.

\section{Acknowledgments}

The authors thank Tahsin Shaikh, Bhopinder Sagoo, and Robeela Begum of Birmingham PCT for their support and work in recruiting participants for this study.

\section{References}

1. Zimmet, P, Shaw J, Alberti KG. Preventing type 2 diabetes and the dysmetabolic syndrome in the real world: a realistic view. Diabet Med. 2003;20:693-702.

2. Jarrett RJ. The cardiovascular risk associated with impaired glucose tolerance. Diabet Med. 1996;13(3 suppl 2):S15-S19.

3. Perry RC, Baron AD. Impaired glucose tolerance: why is it not a disease? Diabetes Care. 1999;22:883-885.

4. Liye H, Lvyun Z, Guangyao S, Luping R. Investigation of early change of endothelial function and related factors in individuals with hyperglycemia. Diabetes Res Clin Pract. 2011;92:194-197.

5. Singleton JR, Smith AG, Russell JW, Feldman EL. Microvascular complications of impaired glucose tolerance. Diabetes. 2003; 52:2867-2873.

6. O'Rourke MF, Safar ME. Relationship between aortic stiffening and microvascular disease in brain and kidney: cause and logic of therapy. Hypertension. 2005;46:200-204.

7. Patel SR, Bellary S, Qin L, et al. Abnormal retinal vascular function and lipid levels in a sample of healthy UK South Asians. Br J Ophthalmol. 2011;95:1573-1576.

8. Heitmar R, Blann A, Cubbidge RP, Lip G, Gherghel D. Continuous retinal vessel diameter measurements-the future of retinal vessel assessment? Invest Ophthalmol Vis Sci. 2010; 51:5833-5839.
9. Caballero AE, Arora S, Saouaf R, et al. Microvascular and macrovascular reactivity is reduced in subjects at risk for type 2 diabetes. Diabetes. 1999;48:1856-1862.

10. Albutt, EC, Nattrass M, Northam BE. Glucose tolerance test and glycosylated haemoglobin measurement for diagnosis of diabetes mellitus - an assessment of the criteria of the WHO Expert Committee on Diabetes Mellitus 1980. Ann Clin Biochem. 1985;22(pt 1):67-73.

11. Sorensen KE, Dorup I, Hermann AP, Mosekilde L. Combined hormone replacement therapy does not protect women against the age-related decline in endothelium-dependent vasomotor function. Circulation. 1998;97:1234-1238.

12. Moens AL, Goovaerts I, Claeys MJ, Vrints CJ. Flow-mediated vasodilation: a diagnostic instrument, or an experimental tool? Chest. 2005;127:2254-2263.

13. Lloyd-Jones DM, Wilson PW, Larson MG, et al. Framingham risk score and prediction of lifetime risk for coronary heart disease. Am J Cardiol. 2004;94:20-24.

14. Gherghel D, Griffiths HR, Hilton EJ, Cunliffe IA, Hosking SL. Systemic reduction in glutathione levels occurs in patients with primary open-angle glaucoma. Invest Ophthalmol Vis Sci. 2005;46:877-883.

15. Blann AD. von Willebrand factor antigen: a radial immunodiffusion method evaluated and compared with an ELISA method. Med Lab Sci. 1992;49:141-143.

16. O'Leary DH, Polak JF, Kronmal RA, et al. Thickening of the carotid wall: a marker for atherosclerosis in the elderlyCardiovascular Health Study Collaborative Research Group. Stroke. 1996;27:224-231.

17. Garhofer G, Bek T, Boehm AG, et al. Use of the retinal vessel analyzer in ocular blood flow research. Acta Ophthalmol. 2010;88:717-722.

18. Nagel E, Vilser W, Lanzl I. Age, blood pressure, and vessel diameter as factors influencing the arterial retinal flicker response. Invest Ophthalmol Vis Sci. 2004;45:1486-1492.

19. Heitmar R, Blann A, Cubbidge R, Lip G, Gherghel D. Continuous retinal vessel diameter measurements-the future of retinal vessel assessment? Invest Ophthalmol Vis Sci. 2010; 51:5833-5839.

20. Corretti MC, Anderson TJ, Benjamin EJ, et al. Guidelines for the ultrasound assessment of endothelial-dependent flowmediated vasodilation of the brachial artery: a report of the International Brachial Artery Reactivity Task Force. J Am Coll Cardiol. 2002;39:257-265.

21. Garhofer G, Zawinka C, Resch H, et al. Reduced response of retinal vessel diameters to flicker stimulation in patients with diabetes. Br J Ophthalmol. 2004;88:887-891.

22. Mandecka A, Dawczynski J, Vilser W, et al. Abnormal retinal autoregulation is detected by provoked stimulation with flicker light in well-controlled patients with type 1 diabetes without retinopathy. Diabetes Res Clin Pract. 2009;86:51-55.

23. Su Y, Liu XM, Sun YM, et al. The relationship between endothelial dysfunction and oxidative stress in diabetes and prediabetes. Int J Clin Pract. 2008;62:877-882.

24. Polak K, Dorner G, Kiss B, et al. Evaluation of the Zeiss retinal vessel analyser. Br J Ophthalmol. 2000;84:1285-1290.

25. Ilanne-Parikka P, Eriksson JG, Lindström J, et al. Effect of lifestyle intervention on the occurrence of metabolic syndrome and its components in the Finnish Diabetes Prevention Study. Diabetes Care. 2008;31:805-807.

26. Hanefeld M, Karasik A, Koehler C, Westermeier T, Chiasson JL. Metabolic syndrome and its single traits as risk factors for diabetes in people with impaired glucose tolerance: the STOPNIDDM trial. Diab Vasc Dis Res. 2009;6:32-37.

27. DeFronzo RA, Abdul-Ghani M. Assessment and treatment of cardiovascular risk in prediabetes: impaired glucose tolerance and impaired fasting glucose. Am J Cardiol. 2011;108(3 suppl):3B-24B. 
28. Tripathy D, Mohanty P, Dhindsa S, et al. Elevation of free fatty acids induces inflammation and impairs vascular reactivity in healthy subjects. Diabetes. 2003;52:2882-2887.

29. Pereira EC, Ferderbar S, Bertolami MC, et al. Biomarkers of oxidative stress and endothelial dysfunction in glucose intolerance and diabetes mellitus. Clin Biochem. 2008;41: 1454-1460.

30. Neri S, Calvagno S, Mauceri B, et al. Effects of antioxidants on postprandial oxidative stress and endothelial dysfunction in subjects with impaired glucose tolerance and type 2 diabetes. Eur J Nutr. 2010;49:409-416.

31. Montagnani M, Golovchenko I, Kim I, et al. Inhibition of phosphatidylinositol 3-kinase enhances mitogenic actions of insulin in endothelial cells. J Biol Cbem. 2002;277:1794-1799.

32. Rask-Madsen C, Li Q, Freund B, et al. Loss of insulin signaling in vascular endothelial cells accelerates atherosclerosis in apolipoprotein E null mice. Cell Metab. 2010;11:379-389.

33. Brohall G, Oden A, Fagerberg B. Carotid artery intima-media thickness in patients with Type 2 diabetes mellitus and impaired glucose tolerance: a systematic review. Diabet Med. 2006;23:609-616.

34. Aydin Y, Berker D, Ustun I, et al. Evaluation of carotid intima media thickness in impaired fasting glucose and impaired glucose tolerance. Minerva Endocrinol. 2011;36:171-179.

35. Wong TY, Barr EL, Tapp RJ, et al. Retinopathy in persons with impaired glucose metabolism: the Australian Diabetes Obesity and Lifestyle (AusDiab) study. Am J Ophthalmol. 2005;140: 1157-1159.

36. Wong TY, Mohamed Q, Klein R, Couper DJ. Do retinopathy signs in non-diabetic individuals predict the subsequent risk of diabetes? Br J Opbthalmol. 2006;90:301-303.
37. Milman S, Crandall JP. Mechanisms of vascular complications in prediabetes. Med Clin North Am. 2011;95:309-325, vii.

38. Nagel E, Vilser W. Flicker observation light induces diameter response in retinal arterioles: a clinical methodological study. Br J Ophthalmol. 2004;88:54-56.

39. Gugleta K, Zawinka C, Rickenbacher I, et al. Analysis of retinal vasodilation after flicker light stimulation in relation to vasospastic propensity. Invest Ophthalmol Vis Sci. 2006;47: 4034-4041.

40. Kotliar KE, Nagel E, Vilser W, Lanzl IM. Functional in vivo assessment of retinal artery microirregularities in glaucoma. Acta Opbthalmologica. 2008;86:424-433.

41. Klijn CJM, Kappelle LJ, van Schooneveld MJ, et al. Venous stasis retinopathy in symptomatic carotid artery occlusion. Stroke. 2002;33:695-701.

42. de Jong FJ, Vernooij MW, Ikram MK, et al. Arteriolar oxygen saturation, cerebral blood flow, and retinal vessel diameters: The Rotterdam Study. Ophthalmology. 2008;115:887-892.

43. Krentz AJ, Clough G, Byrne CD. Interactions between microvascular and macrovascular disease in diabetes: pathophysiology and therapeutic implications. Diabetes Obes Metab. 2007;9:781-791.

44. Delles C, Michelson G, Harazny J, et al. Impaired endothelial function of the retinal vasculature in hypertensive patients. Stroke. 2004;35:1289-1293.

45. Patton N, Aslam T, Macgillivray T, et al. Retinal vascular image analysis as a potential screening tool for cerebrovascular disease: a rationale based on homology between cerebral and retinal microvasculatures. J Anat. 2005;206:319-348. 